\title{
DISTINGUISHED SUBFIELDS
}

BY

\author{
JAMES K. DEVENEY AND JOHN N. MORDESON ${ }^{1}$
}

\begin{abstract}
Let $L$ be a finitely generated nonalgebraic extension of a field $K$ of characteristic $p \neq 0$. A maximal separable extension $D$ of $K$ in $L$ is distinguished if $L \subseteq K^{p^{-n}}(D)$ for some $n$. Let $d$ be the transcendence degree of $L$ over $K$. If every maximal separable extension of $K$ in $L$ is distinguished, then every set of $d$ relatively $p$-independent elements is a separating transcendence basis for a distinguished subfield. Conversely, if $K\left(L^{p}\right)$ is separable over $K$, this condition is also sufficient. A number of properties of such fields are determined and examples are presented illustrating the results.
\end{abstract}

0. Introduction. Let $L$ be a finitely generated extension of a field $K$ of characteristic $p \neq 0$. If $L$ is algebraic over $K$, then there is a unique intermediate field $D$ such that $D$ is separable over $K$ and $L$ is purely inseparable over $D$. If $L$ is not algebraic over $K$, there will still be maximal separable extensions $S$ of $K$ in $L$ and $L$ will necessarily be purely inseparable finite dimensional over any such subfield $S$. However, in general $S$ is far more being unique. If $p^{s}$ is the minimum of the degrees [ $L: S$ ], $s$ is called the order of inseparability of $L / K(\operatorname{inor}(L / K))$. In [5], Dieudonne studied such maximal separable extensions and established that there must be an $S$ such that $L \subseteq K^{p^{-\infty}}(S)$, that is, $L$ can be obtained from $S$ by adjoining $p^{n}$ th roots of elements of $K$. Such a field $S$ is called distinguished. In [7], Kraft established that the distinguished maximal separable intermediate fields are precisely those over which $L$ is of minimal degree. In this paper we examine the question of when every maximal separable intermediate field is distinguished, a property which holds for algebraic extensions.

If $n$ is the least nonnegative integer such that $K\left(L^{p^{n}}\right)$ is separable over $K, n$ is called the inseparability exponent, $\operatorname{inex}(L / K)$. Throughout this paper $n$ will be used to denote inex $(L / K)$ and $d$ will denote the transcendence degree of $L / K$. If $D$ is distinguished for $L / K$, then $K\left(L^{p^{n}}\right)=K\left(D^{p^{n}}\right)$ and hence $L \subseteq K^{p-n}(D)$. Thus, if $Y$ is a relative $p$-basis of $D$ over $K, Y$ is relatively $p$-independent in $L$ over $K$. Since $D$ is separable over $K, Y^{p^{n}}$ is a relative $p$-basis of $K\left(D^{p^{n}}\right)$, i.e. $K\left(L^{p^{n}}\right)$, over $K\left(D^{p^{n+1}}\right)$. Thus $D$ is of the form $K\left(L^{p^{n}}\right)(Y)$. We also note that if $S / K$ is separable and $L / S$ is purely inseparable, $S$ is a maximal separable subfield of $L / K$ if and only if $L^{p} \cap S \subseteq K\left(S^{p}\right)$ [3, Lemma 1.2, p. 46]. $L$ is modular over $K$ if and only if $L^{p^{\prime}}$ and $K$ are linearly disjoint for all $r$. If $L / K$ is finite dimensional purely

Received by the editors March 12, 1979 and, in revised form, August 30, 1979; presented to the Society, November 10, 1979.

AMS (MOS) subject classifications (1970). Primary $12 \mathrm{~F} 15$.

Key words and phrases. Distinguished subfields, modular field extension, irreducible field extension.

${ }^{1}$ This author was supported in part by a faculty summer research grant from Creighton University. 
inseparable, $L / K$ is modular if and only if it is a tensor product of simple extensions [11].

Now suppose the order of inseparability of $L / K$ is $s$. Any intermediate field $L_{1}$ of $L / K$ which also has order of inseparability $s$ will be called a form of $L / K$. In [4, Theorem 1.4, p. 657] it is shown that there exists a unique minimal intermediate field $L^{*}$ of $L / K$ which is a form of $L / K$. The field $L^{*}$ is called the irreducible form of $L / K$ and $L^{*} / K$ is called the irreducible. For example, if $P$ is a perfect field and $\{x, u, v\}$ is algebraically independent over $P$, let $K=P\left(u^{p}, v^{p}\right)$ and $L=K\left(x, u x^{p}+v\right)$. Then $K(x)$ is distinguished, $K\left(u x^{p}+v\right)$ is maximal separable but not distinguished and $K\left(x^{p}, u x^{p}+v\right)$ is a form of $L / K$ (and in fact is the irreducible form).

In §I we develop necessary conditions for every maximal separable subfield of $L / K$ to be distinguished. Theorem 4 establishes the condition that every set of $d$ relatively $p$-independent elements must be a separating transcendence basis for a distinguished subfield. Extensions with this property are then shown to be separable algebraic extensions of irreducible extensions (Theorem 5). In particular, any set of $d$ relatively $p$-independent elements must be algebraically independent over $K$, a property similar to the characterizing property of a separable extension that the elements of any relative $p$-basis must be algebraically independent $[8$, Theorem 11, p. 281].

§II deals exclusively with the inseparability exponent 1 case. It is shown that every maximal separable subfield is distinguished if and only if every set of $d$ relatively $p$-independent elements is a separating transcendence basis for a distinguished subfield. Moreover, if $L / K$ has the property, so does any intermediate field $L_{1} / K$. §III presents examples having the property and indicating why it is necessary to restrict the results of $\S$ II to exponent 1 . §IV develops criteria which force those $L / K$ having every maximal separable subfield distinguished to be of exponent less than or equal to 1 . In fact, we conjecture that this must always be true (in the nonalgebraic case).

\section{Necessary conditions.}

THEOREM 1. If every maximal separable intermediate field of $L / K$ is distinguished, then $K^{p^{-n}}\left(L^{p^{i}}\right) \cap L=K\left(L^{p^{i}}\right)$ for $i=0,1, \ldots$

Proof. The proof is by induction on $i$. The conclusion is immediate for $i=0$. Assume the result for $0 \leqslant i<n$. Suppose $\theta \in K^{p^{-n}}\left(L^{p^{i+1}}\right) \cap L \backslash K\left(L^{p^{i+1}}\right)$ and $\theta$ is transcendental over $K$. Now $\theta \in K^{p^{-n}}\left(L^{p^{i}}\right) \cap L=K\left(L^{p^{i}}\right)$. Since $K(\theta) / K$ is separable, $\theta$ is in a maximal separable intermediate field of $L / K$, say $S$. We show $\theta$ is not in any distinguished intermediate field and hence $S$ is not distinguished, a contradiction. Suppose $\theta \in D$, a distinguished intermediate field. Then

$$
\theta \in D \cap K\left(L^{p^{i}}\right) \subseteq\left(D \otimes_{K} 1\right) \cap\left(K\left(D^{p^{i}}\right) \otimes_{K} K^{p^{-n}}\right)=K\left(D^{p^{i}}\right) .
$$

Now $D=K\left(L^{p^{n}}\right)(Y)$ where $Y$ is relatively $p$-independent in $L / K$ and $Y^{p^{n}}$ is a relative $p$-basis of $K\left(L^{p^{n}}\right) / K$. Thus

$$
\theta \in K\left(L^{p^{n}}\right)\left(Y^{p^{i}}\right) \subseteq K\left(L^{p^{i+1}}\right)\left(Y^{p^{i}}\right) .
$$


Now $Y^{p^{i}}$ is relatively $p$-independent in $K\left(L^{p^{i}}\right) / K$ and since $\theta \notin K\left(L^{p^{i+1}}\right)$, there exists $y \in Y$ such that $\theta \notin K\left(L^{p^{i+1}}\right)\left(Y^{p^{i}} \backslash\left\{y^{p^{i}}\right\}\right)$. Thus

$$
y^{p^{i}} \in K\left(L^{p^{i+1}}\right)\left(Y^{p^{i}} \backslash\left\{y^{p^{i}}\right\}, \theta\right) .
$$

Note that

$$
\begin{aligned}
\theta \in K\left(L^{p^{i}}\right) \cap K^{p^{-n}}\left(L^{p^{i+1}}\right) & \subseteq\left(K^{p^{-n+i}} \otimes_{K} K\left(D^{p^{i}}\right)\right) \cap\left(K^{p^{-n}} \otimes_{K} K\left(D^{p^{i+1}}\right)\right) \\
& =K^{p^{-n+1}} \otimes_{K} K\left(D^{p^{i+1}}\right)
\end{aligned}
$$

and thus $\theta^{p^{n-i}} \in K\left(D^{p^{n+1}}\right)=K\left(L^{p^{n+1}}\right)$. Hence

$$
y^{p^{n}} \in K\left(L^{p^{n+1}}\right)\left(Y^{p^{n}} \backslash\left\{y^{p^{n}}\right\}, \theta^{p^{n-i}}\right)=K\left(L^{p^{n+1}}\right)\left(Y^{p^{n}} \backslash\left\{y^{p^{n}}\right\}\right)
$$

which contradicts the relative $p$-independence of $Y^{p^{n}}$ in $K\left(L^{p^{n}}\right) / K$. Now suppose $\theta$ is algebraic over $K$. Let $t \in K\left(L^{p^{i+1}}\right)$ be transcendental over $K$. Then $\theta+t \in$ $K^{p^{-n}}\left(L^{p^{i+1}}\right) \cap L \backslash K\left(L^{p^{i+1}}\right)$ and is transcendental over $K$. However, this case has been shown to be impossible.

Now assume $K^{p^{-n}}\left(L^{p^{i}}\right) \cap L=K\left(L^{p^{i}}\right)$ for $i \geqslant n$ and let $\theta \in K^{p^{-n}}\left(L^{p^{i+1}}\right) \cap L$. Then $\theta \in K^{p^{-n}}\left(L^{p^{i}}\right) \cap L=K\left(L^{p^{i}}\right)$. Thus

$$
\begin{aligned}
\theta \in K^{p^{-n}}\left(L^{p^{i+1}}\right) \cap K\left(L^{p^{i}}\right) & =\left(K^{p^{-n}} \otimes_{K} K\left(L^{p^{i+1}}\right)\right) \cap\left(1 \otimes_{K} K\left(L^{p^{i}}\right)\right) \\
& =K\left(L^{p^{i+1}}\right)
\end{aligned}
$$

since $K^{p^{-n}} \otimes_{K} K\left(L^{p^{i}}\right)$ is a field. Thus $K^{p^{-n}}\left(L^{p^{i+1}}\right) \cap L=K\left(L^{p^{i+1}}\right)$.

Corollary 2. If $K^{p^{-n}}\left(L^{p^{i}}\right) \cap L=K\left(L^{p^{i}}\right)$, for $i=0,1, \ldots$, then $K^{p^{-n}}\left(K^{p^{i}}\left(L^{p^{j+i}}\right)\right) \cap K\left(L^{p^{j}}\right)=K\left(L^{p^{j+i}}\right)$, for $i=0,1, \ldots$, for any $j$, hence $K\left(L^{p^{j}}\right)$ also has the necessary condition.

Proof. $K^{p^{-n}}\left(K^{p^{i}}\left(L^{p^{j+i}}\right)\right) \cap K\left(L^{p^{j}}\right)=K^{p^{-n}}\left(L^{p^{j+i}}\right) \cap K\left(L^{p^{j}}\right) \subseteq K^{p^{-n}}\left(L^{p^{j+i}}\right) \cap L$ $=K\left(L^{p^{j+i}}\right)$. Clearly $K\left(L^{p^{j+i}}\right) \subseteq K^{p^{-n}}\left(K^{p^{i}}\left(L^{p^{i+j}}\right)\right) \cap K\left(L^{p^{j}}\right)$. Although $K\left(L^{p^{j}}\right)$ will be of inseparable exponent less than that of $L / K$, clearly $K\left(L^{p^{j}}\right)$ has the necessary condition.

Proposition 3. Let $\bar{K}$ be the algebraic closure of $K$ in $L$. If every maximal separable intermediate field of $L / K$ is distinguished, then $\bar{K} / K$ is separable.

Proof. Let $S$ be the maximal separable intermediate field of $\bar{K} / K$ and let $D$ be a maximal separable intermediate field of $L / S$. Since any maximal separable intermediate field of $L / K$ must contain $S, D$ is maximal separable for $L / K$, whence distinguished for $L / K$ and $L / S$. Thus by Theorem $1, S^{p^{-1}} \cap L \subseteq S^{p^{-n}}\left(L^{p^{n}}\right) \cap L$ $=S\left(L^{p^{n}}\right)$, and since $S\left(L^{p^{n}}\right) / S$ is separable, $S^{p^{-1}} \cap L=S$. Thus $S=\bar{K}$.

Theorem 4. Assume every maximal separable intermediate field of $L / K$ is distinguished. Then every set of $d$ relatively p-independent elements is a separating transcendence basis for a distinguished subfield.

Proof. We use induction on $d$. Assume $d=1$ and let $x$ be relatively $p$-independent. Since $\bar{K} / K$ is separable, $x$ is transcendental over $K$. Let $S$ be a maximal separable extension of $K$ in $L$ containing $K(x)$. Then $S$ is distinguished. If $B$ is a 
$p$-basis of $K$, since $x \notin K\left(S^{p}\right)=S^{p}(B), B \cup\{x\}$ is $p$-independent in $S$, i.e., $S$ is separable over $K(x)$. Thus $S / K(x)$ is separable algebraic and hence $x$ is a separating transcendence basis for a distinguished subfield.

Now assume $d>1$ and let $\left\{x_{1}, \ldots, x_{d}\right\}$ be relatively $p$-independent in $L / K$. Then, as above, $x_{1}$ is transcendental over $K$. Since $x_{1}$ is relatively $p$-independent in $L / K$, any maximal separable extension of $K\left(x_{1}\right)$ in $L$ will be a maximal separable extension of $K$ in $L$, hence will be distinguished for $L / K$ and hence for $L / K\left(x_{1}\right)$. Thus every maximal separable intermediate field of $L / K\left(x_{1}\right)$ is distinguished. By induction $\left\{x_{2}, \ldots, x_{d}\right\}$ is a separating transcendence basis for a distinguished subfield of $L / K\left(x_{1}\right)$, and hence $\left\{x_{1}, \ldots, x_{d}\right\}$ is one for $L / K$.

THEOREM 5. If every set of $d$ relatively p-independent elements form a separating transcendence basis for a distinguished subfield, then $L=L^{*}(\theta)$ where $L^{*} / K$ is the irreducible form of $L / K$ and $\theta$ is separable algebraic over $L^{*}$.

Proof. Let $C^{*}$ be the unique intermediate field such that $L / C^{*}$ is separable and $C^{*} / K$ is reliable $\left[2\right.$, Theorem 2.3, p. 141]. If $L \neq C^{*}\left(L^{p}\right)$, choose $L \supseteq L_{1} \supseteq$ $C^{*}\left(L^{p}\right)$ with $\left[L: L_{1}\right]=p$. Since $L_{1} \supseteq C^{*}, L_{1}$ is a form of $L / K[4$, Theorem $1.2, \mathrm{p}$. 656]. Thus $L_{1}$ cannot contain a separating transcendence basis for a distinguished subfield of $L / K$, else the order of inseparability of $L / K$ would be one more than that of $L_{1} / K$. But since $\left[L: L_{1}\right]=p$, and $\left[L: K\left(L^{p}\right)\right] \geqslant p^{d+1}$, at least $d$ elements of $L_{1}$ which are relatively $p$-independent over $K$ must remain $p$-independent in $L$. This contradicts the assumption of the theorem, hence $L=C^{*}\left(L^{p}\right)$, i.e. $L / C^{*}$ is separable algebraic.

Now, if $C^{*} / K$ is not irreducible, then since $C^{*}$ is not separable over any intermediate field of $L / K$ [9, Theorem 1, p. 523], it has a form $L_{0}$ over which $C^{*}$ is purely inseparable and $\left[C^{*}: L_{0}\right]=p$. But now $L=C^{*} \otimes_{L_{0}} S$ where $S / L_{0}$ is separable. Since $L_{0}$ is a form of $L / K, S$ is also a form of $L / K$ and $[L: S]=p$. This leads to a contradiction as above.

Proposition 6. Let $C$ be a subfield of $L / K$ such that $L$ is separable over $C$. If every maximal separable intermediate field of $L / K$ is distinguished, then the same is true for $C / K$.

Proof. Let $D$ be a maximal separable intermediate field of $C / K$. Since $C / D$ is purely inseparable bounded exponent and $L / C$ is separable, $L=F \otimes_{D} C$ for some intermediate field $F$ of $L / D$ such that $F / D$ is separable [6, Proposition 1, p. 302]. Since $L / C$ is separable, $C$ is a form of $L / K$ and hence if $F$ is distinguished for $L / K, D$ is for $C / K$ by a degree argument. Hence it suffices to show $F$ is maximal separable in $L / K$, i.e. $L^{p} \cap F \subseteq K\left(F^{p}\right)$.

But if $b^{p} \in F$, then $b \in\left(D^{p^{-1}} \cap C\right) \otimes_{D} F$ and hence $b^{p} \in\left(C^{p} \cap D\right) \otimes_{D^{p}} F^{p}$ $\subseteq K\left(D^{p}\right)\left(F^{p}\right)=K\left(F^{p}\right)$, so $F$ is maximal separable.

COROLlaRY 7. If every maximal separable intermediate field of $L / K$ is distinguished, then $L$ is a separable algebraic extension of an irreducible extension. 
II. Exponent one. Throughout this section we assume that the inseparability exponent of $L / K$ is 1 . With this restriction, the necessary condition of Theorem 4 is also sufficient.

THEOREM 8. Every maximal separable intermediate field of $L / K$ is distinguished if and only if every set of $d$ relatively p-independent elements is a separating transcendence basis for a distinguished subfield.

Proof. We induct on the order of inseparability of $L / K$. Assume the order of inseparability is 1 and every set of $d$ relatively $p$-independent elements is a separating transcendence basis for a distinguished subfield. By Corollary $7, L=$ $L^{*}(\theta)$ and $L^{*} / K$ is irreducible. Let $S$ be a maximal separable extension of $K$ in $L$. Let $\alpha^{p} \in S$ and $\alpha \notin S$. Then $S(\alpha)$ has order of inseparability 1 , and hence contains $L^{*}$. Thus $L / S(\alpha)$ is separable and purely inseparable and hence $L=$ $S(\alpha)$. Thus $[L: S]=p$ and $S$ is distinguished.

Now assume the order of inseparability is $r>1$ and let $S$ be a maximal separable intermediate field. Consider $S\left(L^{p}\right)(B \backslash b) \equiv L_{0}$ where $B$ is a relative $p$-basis for $L$ over $S$. (Note $r \geqslant 2$ so $|B| \geqslant 2$.) Then $\left[L: L_{0}\right]=p$. Thus $L_{0}$ contains at least $d$ elements which remain $p$-independent in $L$. Hence $L_{0}$ contains a separating transcendence basis for a distinguished subfield of $L / K$ and hence $L_{0} / K$ is not a form of $L / K$. Since we are in exponent $1, L_{0}$ must have one less element in a relative $p$-basis over $K$, and hence every relative $p$-basis for $L_{0} / K$ remains relatively $p$-independent in $L / K$. Thus every set of $d$ elements of a relative $p$-basis for $L_{0} / K$ form a separating transcendence basis for a distinguished intermediate field of $L_{0}$ (since they do for $L$ ), and hence by induction $L_{0}$ has every maximal separable intermediate distinguished. Thus $S$ is distinguished for $L_{0}$ and since the inseparability of $L / K$ is one more than the inseparability of $L_{0} / K, S$ is distinguished for $L / K$.

Lemma 9. Assume every maximal separable intermediate field of $L / K$ is distinguished. If $L_{1}$ is an intermediate field of $L / K$ and $\left[L: L_{1}\right]=p$, then every maximal separable intermediate field of $L_{1} / K$ is distinguished.

Proof. Since $\left[L: L_{1}\right]=p, L / L_{1}$ is separable algebraic or purely inseparable. If $L / L_{1}$ is separable, Proposition 6 applies. Suppose $L / L_{1}$ is purely inseparable. By Corollary 7, $L$ is separable algebraic over its irreducible form, and $L_{1}$ is not a form of $L / K$. Since $L / K$ is of exponent $1, L_{1}$ has one less element than $L$ in a relative $p$-basis over $K$. Since $\left[L: L_{1}\right]=p$, the elements of any relative $p$-basis for $L_{1} / K$ remain relatively $p$-independent in $L / K$. Thus if $d$ is the transcendence degree of $L / K$, any set of $d$ relatively $p$-independent elements of $L_{1} / K$ remain $p$-independent in $L$, hence are a separating transcendence basis for a distinguished subfield of $L$ (Theorem 4 ) which must also be distinguished for $L_{1}$. Thus every maximal separable intermediate field of $L_{1} / K$ is distinguished by Theorem 8 .

THEOREM 10. If $L_{1}$ is an intermediate field of $L / K$ and every maximal separable intermediate field of $L / K$ is distinguished, then the same is true for $L_{1} / K$. 
Proof. $L / L_{1}$ is finitely generated so a finite number of applications of Proposition 6 and Lemma 9 yield the desired result.

III. Examples. We now present some examples to illustrate the results. It should be noted that there is a class of extensions which have every maximal separable intermediate field distinguished. For if $L / K$ is any transcendental extension with order of inseparability 1 , let $L^{*}$ be the irreducible form of $L / K$. If $S$ is a maximal separable extension if $K$ in $L^{*}$ and $\alpha \in L^{*} \backslash S$ with $\alpha^{p} \in S$, then $S(\alpha)$ has order of inseparability 1 , and hence $S(\alpha)=L^{*}$ and $S$ is distinguished. Since all examples in the literature have their irreducible forms of transcendence degree 1 , notably those in [9] and [10], we present the following example.

EXAMPLE 11. There exists a field extension of transcendence degree greater than one which has every maximal separable intermediate field distinguished.

Let $P$ be a perfect field and let $\{v, x, y, z, w\}$ be algebraically independent indeterminates over $P$. Let $K=P(v, x, y)$ and $L=K\left(z, w, z x^{p^{-1}}+w y^{p^{-1}}+v^{p^{-1}}\right)$. Then $L / K$ has transcendence degree 2 and exponent 1 . Let $\left\{b_{1}, b_{2}\right\}$ be relatively $p$-independent in $L / K$. We need to show $\left\{b_{1}, b_{2}\right\}$ is a separating transcendence basis for a distinguished subfield, i.e. $K\left(L^{p}\right)=K\left(L^{p^{2}}\right)\left(b_{1}^{p}, b_{2}^{p}\right)$. By a degree argument, this is true if and only if $K\left(L^{p^{2}}\right)\left(b_{1}^{p}\right) \nsubseteq K\left(L^{p^{2}}\right)\left(b_{2}\right)$ and $K\left(L^{p^{2}}\right)\left(b_{2}^{p}\right)$ $\nsubseteq K\left(L^{p^{2}}\right)\left(b_{1}^{p}\right)$. Thus suppose $K\left(L^{p^{2}}\right)\left(b_{1}^{p}\right) \subseteq K\left(L^{p^{2}}\right)\left(b_{2}^{p}\right)$. Then $L / K\left(L^{p^{2}}\right)\left(b_{2}^{p}\right)$ is modular with a subbasis $b_{1}, b_{2}$ and some $b_{3}$ with exponents $1,1,2$ respectively. We use the method of Sweedler [11, Example 1.1, p. 405] and prove this is impossible by showing the field of constants of all rank $p$ higher derivatives on $L / K$ is $K\left(L^{p}\right)$. Let $d=\left\{d_{0}, d_{1}, \ldots, d_{p}\right\}$ be a rank $p$ higher derivation on $L / K$. Then

$$
\left[d_{1}\left(z x^{p-1}+w y^{p^{-1}}+v^{p^{-1}}\right)\right]^{p}=d_{p}\left(z^{p} x+w^{p} y+v\right)=\left(d_{1}(z)\right)^{p} x+\left(d_{1}(w)\right)^{p} y .
$$

Since $\{1, x, y\}$ is linearly independent over $L^{p}$, we have $d_{p}\left(z^{p}\right)=0=d_{p}\left(w^{p}\right)$. Clearly $d_{i}\left(z^{p}\right)=0=d_{i}\left(w^{p}\right), i=1, \ldots, p-1$. Hence $K\left(L^{p}\right)$ is the field of constants as claimed. Thus any 2 relatively $p$-independent elements are a separating transcendence basis for a distinguished subfield, and hence by Theorem $8, L / K$ has every maximal separable intermediate field distinguished.

EXAMPLE 12. We show the converse of Theorem 5 is not true. Let $P$ be a perfect field and let $\left\{w, x, y_{1}, y_{2}, z\right\}$ be algebraically independent indeterminates over $P$. Let $K=P\left(x, y_{1}, y_{2}\right)$ and $L=K\left(z, w, x^{p^{-1}} z+y_{1}^{p^{-1}}, x^{p^{-1}} w+y_{2}^{p^{-1}}\right)$. Let $L^{*}$ be the irreducible form of $L / K$. If $L=L^{*}\left(L^{p}\right)$, then $L$ is separable algebraic over $L^{*}$, as desired.

If $L \neq L^{*}\left(L^{p}\right)$, then there is a subfield $L_{1}$ over which $L$ is purely inseparable and [ $\left.L: L_{1}\right]=p$ and $L_{1}$ has order of inseparability 2 . We show this is impossible. Such a field $L_{1}$ is of the form $K\left(L^{p}\right)\left(b_{1}, b_{2}, b_{3}\right)$ where $\left\{b_{1}, b_{2}, b_{3}\right\}$ is relatively $p$-independent in $L / K$. If $K\left(L^{p}\right)=K\left(L^{p^{2}}\right)\left(b_{1}^{p}, b_{2}^{p}, b_{2}^{p}\right)$, then $L_{1} / K$ would have order of inseparability 1 , a contradiction. If $K\left(L^{p}\right) \neq K\left(L^{p^{2}}\right)\left(b_{1}^{p}, b_{2}^{p}, b_{3}^{p}\right)$, then since $\left[K\left(L^{p}\right)\right.$ : $\left.K\left(L^{p^{2}}\right)\right]=p^{2}, K\left(L^{p^{2}}\right)\left(b_{1}^{p}, b_{2}^{p}, b_{3}^{p}\right) \subseteq K\left(L^{p^{2}}\right)\left(b_{3}^{p}\right)$ say. But now since $\left[L: K\left(L^{p^{2}}\right)\right]=p^{6}$ and is of exponent $2, L / K\left(L^{p^{2}}\right)\left(b_{3}\right)$ is modular with a subbasis $b_{1}, b_{2}$ and some $b_{4}$ with exponents 1, 1, 2 respectively. But as in Example 11, the field of constants of 
all rank $p$ higher derivatives on $L / K$ is $K\left(L^{p}\right)$, and we have a contradiction. Thus $L=L^{*}(\theta)$ where $L^{*} / K$ is irreducible and $\theta$ is separable algebraic over $L^{*}$. However, it is clear that $\left\{z, x^{p^{-1}} z+y_{1}^{p^{-1}}\right\}$ is not a separating transcendence basis for a distinguished subfield.

EXAMPLE 13. We show that the exponent 1 restriction is essential to Theorem 8 . Let $P$ be a perfect field and let $\{x, y, z\}$ be algebraically independent indeterminates over $P$. Let $K=P(x, y)$ and $L=K\left(z, z x^{p^{-2}}+y^{p^{-2}}\right)$. It is straightforward that $L / K\left(L^{p^{3}}\right)$ is not modular. Thus if $b$ is $p$-independent in $L / K, b^{p^{2}} \notin$ $K\left(L^{p^{3}}\right)$, i.e. $b$ is a separating transcendence basis for a distinguished subfield. Thus every set of 1 relatively $p$-independent element is a separating transcendence basis for a distinguished subfield. However, let $S=K\left(\left(z^{p}+x\left(z^{p} x^{p^{-1}}+y^{p^{-1}}\right)\right)\right)$. Then $[L: S]=p^{3}$ and $L / S$ is not modular. Thus $\left[S^{p^{-1}} \cap L: S\right]=p$. Clearly $S$ is distinguished in $K\left(L^{p}\right) / K$ and since $\left[S^{p^{-1}} \cap K\left(L^{p}\right): S\right]=p, L^{p} \cap S=\left(K\left(L^{p}\right)\right)^{p}$ $\cap S \subseteq K\left(S^{p}\right)$, i.e. $S$ is a maximal separable extension of $K$ in $L$. Since $[L: S]=p^{3}$ and the order of inseparability of $L / K$ is $p^{2}, S$ is not distinguished.

IV. Restrictions for exponent one. In this section we develop results which force an extension $L / K$ which has every maximal separable subfield distinguished to have inseparability exponent 1.

LEMMA 14. Suppose the transcendence degree of $L$ over $K$ is one. If $K^{p^{-n}}\left(L^{p}\right) \cap L$ $=K\left(L^{p}\right)$, then every maximal separable intermediate field is either distinguished or contained in $K\left(L^{p}\right)$.

Proof. Let $D$ be maximal separable and let $x$ be a separating transcendence basis for $D / K$. If $x \in K\left(L^{p}\right)$, then since $D$ is separable over $K(x), D \subseteq K\left(L^{p}\right)$. If $x \notin K\left(L^{p}\right)$, then $x \notin K^{p^{-n}}\left(L^{p}\right)$. Thus $x^{p^{n}} \notin K\left(L^{p^{n+1}}\right)$ and the separable algebraic closure of $K(x)$, i.e. $D$, is a distinguished subfield.

Proposition 15. Suppose the transcendence degree of $L$ over $K$ is 1 and every maximal separable intermediate field is distinguished. Then:

(1) every maximal separable intermediate field of $K\left(L^{p}\right) / K$ is distinguished or contained in $K\left(L^{p^{i+1}}\right)$;

(2) every maximal separable intermediate field of $K\left(L^{p^{n-1}}\right)$ is distinguished.

Proof. (1) follows from Theorem 1 and Lemma 14. For (2), if $D$ is distinguished for $K\left(L^{p^{n-1}}\right) / K, K\left(D^{p}\right)=K\left(L^{p^{n}}\right)$, and hence a maximal separable extension of $K$ in $K\left(L^{p^{n-1}}\right)$ cannot be contained in $K\left(L^{p^{n}}\right)$.

$L$ is a finite dimensional purely inseparable extension of $K\left(L^{p n}\right)$. We let $L_{m}$ denote the unique minimal purely inseparable extension of $L$ such that $L_{m} / K\left(L^{p^{m}}\right)$ is modular [11, Theorem 6, p. 408]. Note that $L_{m} / K\left(L^{p^{n}}\right)$ is also of exponent $n$.

THEOREM 16. Suppose every maximal separable intermediate field of $L / K$ is distinguished. Then $K^{p^{-1}} \nsubseteq L_{m}$ if and only if $\operatorname{in} \operatorname{ex}(L / K)=1$.

Proof. Suppose $K^{p^{-1}} \nsubseteq L_{m}$ and $\operatorname{inex}(L / K)=n \geqslant 2$ Let $X$ be a set of $d-1$ relatively $p$-independent elements of $L / K$. Then $X$ is part of a separating transcendence basis for a distinguished subfield by Theorem 4. Also, since $X$ is relatively 
$p$-independent, any maximal separable subfield for $L / K(X)$ is one for $L / K$ and hence every maximal separable subfield of $L / K(X)$ is distinguished. Now $X$ is a subbasis of $K\left(L^{p^{n}}\right)(X) / K\left(L^{p^{n}}\right)$ and since every element of $X$ has maximal exponent, $X$ is part of a subbasis of $L_{m} / K\left(L^{p^{n}}\right)$. Thus $L_{m} / K\left(L^{p^{n}}\right)(X)$ is modular.

Let $k \in K \backslash L_{m}^{p}, z \in L \backslash K\left(L^{p}\right)(X)$, and $w \in L \backslash K\left(L^{p}\right)(X, z)$ such that $w$ has exponent $n$ over $K\left(L^{p^{n}}\right)(X, z)$. Such a $w$ exists because $K\left(L^{p^{n}}\right)(X, z)$ is distinguished and inex $(L / K)=n$. Set $D=K\left(L^{p^{n}}\right)(X)\left(z^{p}+k w^{p}\right)$. Since $D \subseteq$ $K\left(L^{p}\right)(X), D$ is not distinguished in $L / K(X)$. We show $D$ is maximal separable in $L / K(X)$ and hence have a contradiction to $n \geqslant 2$.

Clearly $D / K(X)$ is separable and $L / D$ is purely inseparable. Thus it suffices to show $L^{p} \cap D \subseteq K(X)\left(D^{p}\right)\left[3\right.$, Lemma 1.2, p. 46]. We first calculate $K(X)\left(D^{p}\right)$. $D / K\left(L^{p^{n}}\right)(X)$ has exponent $n-1$ since $X \cup\{z, w\}$ is part of a subbasis of

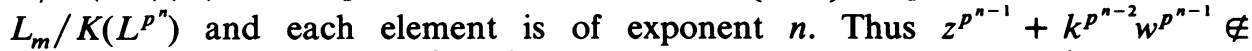
$K\left(L^{p^{n}}\right)(X)$, so $z^{p^{n-1}}+k^{p^{n-2} w^{p^{n-1}}}$ is in a relative $p$-basis of $K\left(L^{p^{n-1}}\right)(X) / K(X)$. Since every maximal separable subfield of $L / K(X)$ is distinguished, the same is true for $K\left(L^{p^{n-1}}\right)(X) / K(X)$ by Lemma 14. Thus $z^{p^{n-1}}+k^{p^{n-2}} w^{p^{n-1}}$ is a separating transcendence basis for a distinguished subfield of $K\left(L^{p^{n-1}}\right)(X) / K(X)$. Therefore

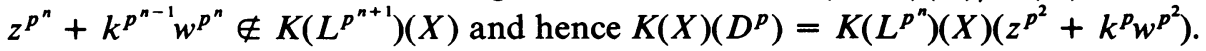

Now suppose $L^{p} \cap D \nsubseteq K(X)\left(D^{p}\right)$, i.e. there exists $c \in L$ such that $c^{p} \in$ $D \backslash K(X)\left(D^{p}\right)$. Then $D=K\left(L^{p^{n}}\right)(X)\left(c^{p}\right)$ and $c$ must have exponent $n$ over $K\left(L^{p^{n}}\right)(X)$. Thus $X \cup\{c\}$ is part of a subbasis of $L_{m} / K\left(L^{p^{n}}\right)$ and hence $L_{m} / D$ is modular. But, using the same methods as in Example 11 , if $L_{m} / D$ is modular, $z^{p}$ and $w^{p}$ are in $D$ and $\left[D: K\left(L^{p^{n}}\right)(X)\right]>p^{n-1}$, a contradiction.

Conversely, if inex $(L / K)=1, L_{m}=L$ and Proposition 3 shows $K^{p^{-1}} \nsubseteq L$.

COROLlary 17. Suppose every maximal separable intermediate field of $L / K$ is distinguished. If any of the conditions below hold, then $\operatorname{in} \operatorname{ex}(L / K) \leqslant 1$;

(a) $L / K\left(L^{p^{n}}\right)$ is modular;

(b) $\left[L: K\left(L^{p}\right)\right]=p^{d+1}$;

(c) $\left[K: K^{p}\right]=p^{e}$ where $e=0,1,2$, or $\infty$.

Proof. If $L / K$ is separable, the result is trivial. Thus assume $L / K$ is inseparable. By Proposition 3, $K^{p^{-1}} \cap L=K$. If $L / K\left(L^{p^{n}}\right)$ is modular, $L=L_{m}$ and $K^{p^{-1}} \nsubseteq L_{m}$. By Theorem 16, $\operatorname{in} \operatorname{ex}(L / K)=1$. If $\left[L: K\left(L^{p}\right)\right]=p^{d+1}$, then it follows that $L / K\left(L^{p^{n}}\right)$ is modular [1, Theorem 22, p. 1308]. If $\left[K: K^{p}\right]<2, L / K\left(L^{p^{n}}\right)$ is modular [1, Corollary 2.3 , p. 1308]. If $\left[K: K^{p}\right]=\infty$, since $\left[L_{m}: L\right]<\infty, K^{p^{-1}} \nsubseteq L_{m}$.

\section{REFERENCES}

1. J. Deveney and J. Mordeson, Subfields and invariants of inseparable field extensions, Canad. J. Math. 29 (1977), 1304-1311.

2. Invariants of reliable field extensions, Arch. Math. 29 (1977), 141-147.

3. __ Splitting and modularly perfect fields, Pacific J. Math. 83 (1979), 45-54.

4. _ The order of inseparability of fields, Canad. J. Math. 31 (1979), 655-662.

5. J. Dieudonne, Sur les extensions transcendentes, Summa Brasil. Math. 2 (1947), 1-20. MR 10, 5.

6. N. Heerema, and D. Tucker, Modular field extensions, Proc. Amer. Math. Soc. 53 (1975), 301-306. MR 53 \#5551. 
7. H. Kraft, Inseparable Korperweiterungen, Comment. Math. Helv. 45 (1970), 110-118. MR 41 \#5333.

8. S. Mac Lane, Modular fields. I. Separating transcendency bases, Duke Math. J. 5 (1939), 272-293.

9. J. Mordeson and B. Vinograde, Relatively separated transcendental field extensions, Arch. Math. (Basel) 24 (1973), 521-526. MR 51 \# 12807.

10. Inseparable embeddings of separable transcendental extensions, Arch. Math. (Basel) 26 (1975), 606-610. MR 35 \#2913.

11. M. Sweedler, Structure of inseparable extensions, Ann. of Math. (2) 87 (1968), 401-410; correction, ibid. (2) 89 (1969), 206-207. MR 36 \#6391; 38 \#4451.

Department of Mathematical Sciences, Virginia Commonwealth University, Richmond, VIRGINIA 23284

Department of Mathematics, Creighton University, Omaha, Nebraska 68131 